\title{
Cerebral Haemorrhage in a Young Male due to Idiopathic Unilateral Sigmoid and Transverse Sinus Thrombosis, Treated with Anticoagulation
}

\author{
Sharjeel Shaikh, Zulfiqar Ali Sandhu* and Muhammad Siddique \\ Department of General Medicine, Wexford General Hospital, Ireland
}

Correspondence should be addressed to Zulfiqar Ali Sandhu, Zulfiqar.Sandhu@hse.ie

Received: June 24, 2020; Accepted: July 06, 2020; Published: July 13, 2020

\begin{abstract}
Cerebral venous sinus thrombosis is a rare but serious disorder. Most cases are linked to thrombophilias, pregnancy, infection and malignancy. It has a remarkably varied clinical presentation and is challenging to diagnose. It can be complicated by haemorrhage which makes the treatment decisions more challenging and difficult. Excellent functional recovery is likely with early recognition and treatment of the underlying aetiology.
\end{abstract}

\section{KEYWORDS}

Cerebral venous sinus thrombosis; Haemorrhage; Heparin; Seizure

\section{CASE PRESENTATION}

We present case of 40-year-old gentleman who presented to Wexford General Hospital with history of unilateral left sided occipital headache for a week. Headache was initially occasional, relieved with simple analgesia but became persistent and progressive. He denied having fever, photophobia, visual disturbance and sensory or motor symptoms. Patient had no significant past medical history of note. He denied any addiction or drug misuse. On clinical examination, he had no focal neurological deficit and no papilloedema. His Chest X-Ray, ECG and Routine Bloods (FBC, CRP, U\&E, LFT's and Coagulation studies) were unremarkable. CT brain was organised and Lumbar Puncture was planned for CSF study to look for xanthochromia. While awaiting CT brain he had a witnessed generalised tonic clonic seizure with tongue biting. The seizure activity lasted for two minutes followed by post-ictal confusion of 45 minutes. He underwent a CT Brain with contrast which revealed a small area of left-sided cerebral haemorrhage with possible extension into the adjacent subarachnoid space at the posterior aspect of the adjacent sylvian fissure and non-specific thickening of the lateral aspect of the left tentorium cerebelli with normal paranasal sinuses, mastoid air cells and middle ears (Figure 1). In view of the imaging findings, intracranial CT angiogram and venogram was performed which revealed left sigmoid and transverse sinus thrombosis (Figure 2A - Figure 2C).

He was commenced on anti-epileptic and low molecular weight heparin (tinzaparin 175 IU/kg OD subcutaneously). He was monitored in high dependency unit with hourly neuro-observation. Neurosurgical and

Citation: Sharjeel Shaikh, Cerebral Haemorrhage in a Young Male due to Idiopathic Unilateral Sigmoid and Transverse Sinus Thrombosis, Treated with Anticoagulation. J Clin Cases Rep 3(S3): 3-5. 
neurology advice was obtained in management of this gentleman. He was not for any neurosurgical intervention at that stage. Patient remained well over next 48 hours with GCS-15/15, no neuro-deficit and headache improved. A repeat CT scan was obtained which showed no evolution or change in size of haemorrhage. He was then commenced on warfarin bridging with LMWH to achieve a target INR range 2.0 - 3.0. He remained well over the course of his admission and was discharged with a neurology outpatient follow up. MRI brain venogram performed at three months revealed resolution of both haemorrhage and thrombosis, antiepileptic medication was discontinued at that stage. He was advised to stay on OAC for total duration of 6 months. Thrombophilia screen performed after discontinuation of oral anticoagulation was negative.

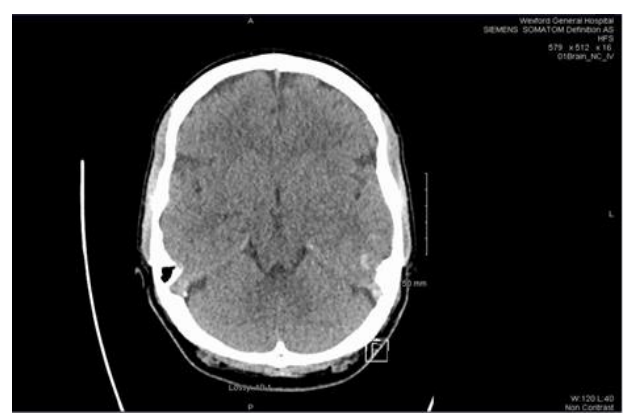

Figure 1: Small area of left-sided cerebral haemorrhage.

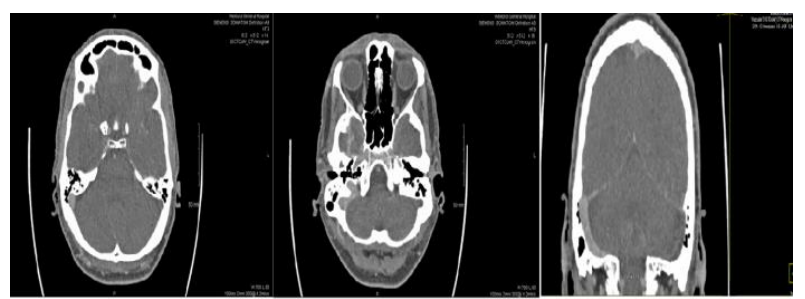

Figure 2: Intracranial CT angiogram and venogram was performed which revealed Left sigmoid and transverse sinus thrombosis.

\section{DISCUSSION}

Cerebral venous sinus thrombosis is rare and life threatening condition with mortality ranging 2 percent 10 percent. According to "The International Study on Cerebral Vein and Dural Sinus" thrombosis the incidence of cerebral venous sinus thrombosis (CVST) was three times greater in women than men, $78 \%$ of all cases were aged less than 50 years, and the most common affected site was transverse sinus followed by Superior and Straight Sinus [1]. Risk factors for cerebral venous thrombosis are pregnancy, puerperium, connective tissue disease, malignancy, contraceptive use, trauma, thrombophilia, infections such as sinusitis, otitis and mastoiditis [2]. The most frequent symptoms and signs are headache $(95 \%)$, focal seizures with or without secondary generalization $(47 \%)$, unilateral or bilateral paresis $(43 \%)$ and papilledema $(41 \%)$ [3]. In patients with CVST, spontaneous intracranial haemorrhage accounted for $30 \%$ to $40 \%$ of ICH. CVST-induced ICH includes simple cerebral haemorrhage and venous infarction haemorrhage. The blockade of venous sinuses cause increase venous and capillary pressure results in diapedesis of red blood cells and subsequent rupture of small vessels cause haemorrhage, ICH may be an extension of this sequence of events [4].

The first-line management is systemic anticoagulation, however in cases with subdural haematoma or cerebral bleed the initiation of therapeutic anticoagulation must be carefully weighed against the potential risk of intracranial haemorrhage. Khatib et al. suggested starting immediate anticoagulation if the patient is stable and has no mass effect or midline shift in neuroimaging [5]. The current literature suggests that Low molecular weight heparin (LMWH) may be superior to unfractionated heparin (UFH) in the treatment of CVST. LMWH is frequently associated with a decreased risk of thrombotic complications, major bleeding, and death compared with UFH [6]. If the aetiology of thrombosis is idiopathic or caused by thrombophilia the recommended duration of anticoagulation is 6 months to 12 months. In patients with 1 episode of CVST and transient risk factor such as meningitis or trauma, oral anticoagulation therapy (OAC) should continue for at least 3 months. Lifelong OAC therapy is recommended in patients with 1 episode of CVST and a severe form of thrombophilia or in patients with 2 or more episodes of CVST [7]. 
The evidence for endovascular treatment for CVST is very limited to small case reports and is not recommended as standard treatment algorithm. The indications for surgical intervention in CVST are limited to large haemorrhagic infarcts or subdural haematomas causing significant mass effect and or hydrocephalus [8].

Twelve percent of patients suffer a recurrence of CVST and $14 \%$ a different form of venous thrombosis. The incidence of recurrence is higher in male compared to female with Idiopathic CVST. Seizures rarely occur beyond the acute stages [9].

\section{CONCLUSION}

1. CVST is rare and fatal medical condition. Timely diagnosis and treatment has significant impact on morbidity and mortality.
2. Clinical presentation varies significantly, can present as intracranial haemorrhage. Treatment of complicated CVST with haemorrhage is challenging as there is significant risk of increased bleeding and raised intracranial tension requiring craniotomy.

3. The guidelines regarding treatment duration and choice of anticoagulation are unclear and further studies are needed.

4. This case further supports use of LMWH instead of unfractionated heparin in treatment of CVST even in case of small haemorrhage. Currently Enoxaparin (BD) and Tinzaparin (OD) are the LMWH used for VTE treatment, but no clear benefits or disadvantages have been studied in CVST of either.

\section{REFERENCES}

1. Ferro JM, Canhão P, Stam J, et al. (2004) Prognosis of cerebral vein and dural sinus thrombosis: Results of the International study on cerebral vein and dural sinus thrombosis (ISCVT). Stroke 35(3): 664-670.

2. Park DS, Moon CT, Chun YI, et al. (2014) Clinical characteristics of cerebral venous thrombosis in a single center in Korea. Journal of Korean Neurosurgical Society 56(4): 289-294.

3. De Bruijn SFTM, De Haan RJ, Stam J (2001) Clinical features and prognostic factors of cerebral venous sinus thrombosis in a prospective series of 59 patients. Journal of Neurology, Neurosurgery \& Psychiatry 70(1): 105-108.

4. Girot M, Ferro JM, Canhão P, et al. (2007) Predictors of outcome in patients with cerebral venous thrombosis and intracerebral hemorrhage. Stroke 38(2): 337-342.

5. Khatib K, Baviskar A (2016) Treatment of cerebral venous sinus thrombosis with subdural hematoma and subarachnoid hemorrhage. Journal of Emergencies, Trauma and Shock 9(4): 155-156.

6. AG van Den Belt, Prins MH, Lensing AW, et al. (2000) Fixed dose subcutaneous low molecular weight heparins versus adjusted dose unfractionated heparin for venous thromboembolism. The Cochrane Database of Systematic Reviews 2: CD001100.

7. Demchuk A, Venegas-Torres J (2015) Cranial venous sinus thrombosis diagnosis and management. In Hamilton M, Golfinos J, Pineo G (Eds.): Handbook of bleeding and coagulation for neurosurgery. New York: Thieme: 184-196.

8. Silvis SM, De Sousa DA, Ferro JM, et al (2017) Cerebral venous thrombosis. Nature Reviews Neurology 13(9): 555565.

9. Preter M, Tzourio C, Ameri A, et al. (1996) Long-term prognosis in cerebral venous thrombosis: Follow-up of 77 patients. Stroke 27(2): 243-246. 